\title{
The Existence of Positive Solutions for Fractional Differential Equations with Integral and Disturbance Parameter in Boundary Conditions
}

\author{
Mei Jia and Xiping Liu \\ College of Science, University of Shanghai for Science and Technology, Shanghai 200093, China \\ Correspondence should be addressed to Mei Jia; jiamei-usst@163.com
}

Received 13 November 2013; Accepted 17 January 2014; Published 24 March 2014

Academic Editor: Paul Eloe

Copyright (C) 2014 M. Jia and X. Liu. This is an open access article distributed under the Creative Commons Attribution License, which permits unrestricted use, distribution, and reproduction in any medium, provided the original work is properly cited.

We study the existence and nonexistence of the positive solutions for the integral boundary value problem of the fractional differential equations with the disturbance parameter $a$ in the boundary conditions and the impact of the disturbance parameter $a$ on the existence of positive solutions. By using the upper and lower solutions method, fixed point index theory and the Schauder fixed point theorem, we obtain sufficient conditions for that the problem has at least one positive solution, two positive solutions and no solutions. Under certain conditions, we also obtain the demarcation point which divides the disturbance parameters into two subintervals such that the boundary value problem has positive solutions for the disturbance parameter in one subinterval while no positive solutions in the other.

\section{Introduction}

In this paper, we are concerned with the existence and nonexistence of positive solutions for the boundary value problem of the fractional differential equations

$$
\begin{gathered}
-{ }^{C} D^{\delta} u(t)=f(t, u(t)), \quad t \in J, \\
m_{1} u(0)-n_{1} u^{\prime}(0)=0, \\
m_{2} u(1)+n_{2} u^{\prime}(1)=\int_{0}^{1} g(s) u(s) d s+a,
\end{gathered}
$$

where $J=[0,1], 1<\delta \leq 2, f \in C\left(J \times \mathbb{R}^{+}, \mathbb{R}^{+}\right), m_{i} \geq 0, n_{i} \geq$ $0, m_{i}^{2}+n_{i}^{2}>0, i=1,2, g \in C\left(J, \mathbb{R}^{+}\right)$, disturbance parameter $a \in \mathbb{R}^{+}$, and ${ }^{C} D^{\delta}$ is the Caputo fractional derivative of order $\delta$.

In the recent decades, since fractional differential equations have been applied widely and successfully in the description of complex dynamics, they have been regarded as a valuable tool being used in the fields to handle viscoelastic, physics, chemistry, electrical engineering, biology aspects, and so forth; see [1-12] and references therein. And besides, the boundary value problems for the differential equations appear in many applications; see [13-16]. As a result, the boundary value problems for the fractional differential equations are one of the most active fields in the researches of nonlinear differential equations theories and plenty of meaningful achievements have been gained in the related fields; see $[1-8,17-29]$ and the references therein. Due to that the boundary value problems with the integral boundary conditions include two-, three-, and multipoint boundary value problems as special cases and they can better describe the actual phenomenon; more and more emphases have been put on the researches of them; see $[19,20,30-34]$ and the references therein.

At the same time, while using the methods of the differential equations to solve the actual problems, it is inevitable that there always exists disturbance which will have great influence on the existence of the solutions. In paper [35, 36], the authors studied nonlinear nonlocal boundary value problem with nonhomogeneous boundary conditions

$$
\begin{gathered}
u^{\prime \prime}(t)+f\left(t, u(t), u^{\prime}(t)\right)=0, \quad t \in(0,1), \\
u(0)-\sum_{i=1}^{m} a_{i} u\left(t_{i}\right)=\lambda_{1},
\end{gathered}
$$




$$
u(1)-\sum_{i=1}^{m} b_{i} u\left(t_{i}\right)=\lambda_{2}
$$

where they discussed the impact of disturbance parameters $\lambda_{1}, \lambda_{2}$ on the existence of the solution and obtained some meaningful conclusions. And then, the authors further studied the 2 nth order nonlinear nonlocal boundary value problem with nonhomogeneous boundary conditions; see [37].

The purpose of this paper is to study the impact of the disturbance parameter $a$ on the existence of positive solutions and obtain sufficient conditions for the boundary value problem (1) to have at least one positive solution, at least two solutions, and no solutions. Under certain conditions, we obtain that there exists a constant $a^{*}>0$, which separates $\mathbb{R}^{+}$ into two disjoint subintervals $M=\left[0, a^{*}\right]$ and $N=\left(a^{*},+\infty\right)$ such that the boundary value problem (1) has at least two positive solutions for each $a \in\left(0, a^{*}\right)$, at least one positive solution for $a=0$ and $a=a^{*}$, and no positive solutions for $a \in N$. The main tools we applied are the upper and lower solutions method, fixed point index theory, and the Schauder fixed point theorem.

This paper is organized as follows. In Section 2, we introduce the basic definitions and the basic properties of the integral kernel. In Section 3, we study the comparison principles and the basic lemmas. In Section 4, we consider the existence and nonexistence of the positive solutions of the boundary value problem (1), and we study the impact of the disturbance parameter $a$ on the existence of positive solutions.

\section{Preliminaries}

In this section, we give some basic definitions and lemmas which play an important role in our research.

Definition 1 (See $[2,3]$ ). Let $\delta>0$, for a function $u$ : $(0,+\infty) \rightarrow \mathbb{R}$. The Riemann-Liouville fractional integral operator of order $\delta$ of $u$ is defined by

$$
I^{\delta} u(t)=\frac{1}{\Gamma(\delta)} \int_{0}^{t}(t-s)^{\delta-1} u(s) d s,
$$

provided the integral exists.

The Caputo derivative of order $\delta$ for a function $u$ : $(0,+\infty) \rightarrow \mathbb{R}$ is given by

$$
{ }^{C} D^{\delta} u(t)=\frac{1}{\Gamma(n-\alpha)} \int_{0}^{t} \frac{u^{(n)}(s)}{(t-s)^{\delta+1-n}} d s,
$$

provided the right side is pointwise defined on $(0,+\infty)$, where $n=[\delta]+1$ and $n-1<\delta<n$.

$$
\text { If } \delta=n \text {, then }{ }^{C} D^{\delta} u(t)=u^{(n)}(t) \text {. }
$$

Definition 2. Let $A C[0,1]$ be the space of functions $u$ which are absolutely continuous on $[0,1]$. We denote $A C^{n}[0,1]$ by the set of functions $u$ which have continuous derivatives up to order $n-1$ on $[0,1]$ such that $u^{(n-1)} \in A C[0,1]$. In particular, $A C^{1}[0,1]=A C[0,1]$.
Lemma 3 (See [2]). If $u \in A C^{n}[0,1]$, then the Caputo fractional derivative ${ }^{C} D^{\delta} u(t)$ exists almost everywhere on $[0,1]$, where $n$ is the smallest integer greater than or equal to $\delta$.

Lemma 4 (See [2]). Suppose $\delta>0$ and $u \in A C^{n}(J)$. Then

$$
\begin{gathered}
I^{\delta C} D^{\delta} u(t)=u(t)+c_{0}+c_{1} t+c_{2} t^{2}+\cdots+c_{n-1} t^{n-1}, \\
c_{k}=\frac{u^{(k)}(0)}{k !}, \quad k=0,1,2, \ldots, n-1,
\end{gathered}
$$

where $n$ is the smallest integer greater than or equal to $\delta$.

Definition 5. One says $u$ is a solution of the boundary value problem (1) if $u \in A C^{2}(J),{ }^{C} D^{\delta} u(t) \in C(J)$ and satisfies (1). One says $u$ is a positive solution of the boundary value problem (1) if $u$ is a solution of the boundary value problem (1) and $u(t) \geq 0$ and $u(t) \not \equiv 0$ for $t \in J$.

Throughout this paper, we assume the following conditions hold.

(H1) $\rho>0, \bar{g}>0$ and $n_{2}\left((\delta-1) n_{1}-(2-\delta) m_{1}\right)>0$, where

$$
\begin{aligned}
& \rho=m_{1} m_{2}+m_{1} n_{2}+m_{2} n_{1}, \\
& \bar{g}=\frac{1}{\rho-\int_{0}^{1}\left(n_{1}+m_{1} s\right) g(s) d s} .
\end{aligned}
$$

(H2) There exists a function $c \in A C^{2}(J,(-\infty, 0])$ and ${ }^{C} D^{\delta} c \in C(J)$ such that $m_{1} c(0)-n_{1} c^{\prime}(0)=0, m_{2} c(1)+$ $n_{2} c^{\prime}(1) \geq-1$ and $\int_{0}^{1} g(s) c(s) d s \leq-2$.

(H3) $f(\cdot, u)$ is monotonically increasing with respect to $u$ and $f \not \equiv 0$.

For $y \in C(J)$, firstly we consider the boundary value problem

$$
\begin{gathered}
-{ }^{C} D^{\delta} u(t)=y(t), \quad t \in J, \\
m_{1} u(0)-n_{1} u^{\prime}(0)=0, \\
m_{2} u(1)+n_{2} u^{\prime}(1)=\int_{0}^{1} g(s) u(s) d s .
\end{gathered}
$$

Lemma 6. Suppose (H1) holds. Then the boundary value problem (7) has the unique solution

$$
u(t)=\int_{0}^{1} H(t, s) y(s) d s
$$

where the function $H$ is given by

$$
H(t, s)=G(t, s)+h(t, s),
$$


where

$G(t, s)$

$$
\begin{aligned}
& =\frac{1}{\rho \Gamma(\delta)} \\
& \times\left\{\begin{array}{rr}
\left(n_{1}+m_{1} t\right)\left(m_{2}(1-s)^{\delta-1}+n_{2}(\delta-1)(1-s)^{\delta-2}\right) \\
-\rho(t-s)^{\delta-1}, & 0 \leq s<t \leq 1, \\
\left(n_{1}+m_{1} t\right)\left(m_{2}(1-s)^{\delta-1}+n_{2}(\delta-1)(1-s)^{\delta-2}\right), & 0 \leq t \leq s<1,
\end{array}\right.
\end{aligned}
$$

$$
h(t, s)=\bar{g}\left(n_{1}+m_{1} t\right) \int_{0}^{1} g(r) G(r, s) d r .
$$

Proof. According to Lemma $4,-{ }^{C} D^{\delta} u(t)=y(t)$ is equivalent to the following equation:

$$
u(t)=-I^{\delta} y(t)+c_{0}+c_{1} t
$$

where $c_{0}=u(0), c_{1}=u^{\prime}(0)$.

By the boundary condition $m_{1} u(0)-n_{1} u^{\prime}(0)=0$ and $m_{2} u(1)+n_{2} u^{\prime}(1)=\int_{0}^{1} g(s) u(s) d s$, we can show

$$
m_{1} c_{0}-n_{1} c_{1}=0
$$

$$
\begin{aligned}
& m_{2} c_{0}+\left(m_{2}+n_{2}\right) c_{1} \\
& =\int_{0}^{1} g(s) u(s) d s+m_{2} I^{\delta} y(1)+n_{2} I^{\delta-1} y(1) .
\end{aligned}
$$

Hence, we can obtain

$$
\begin{aligned}
& c_{0}=\frac{n_{1}}{\rho}\left(\int_{0}^{1} g(s) u(s) d s+m_{2} I^{\delta} y(1)+n_{2} I^{\delta-1} y(1)\right), \\
& c_{1}=\frac{m_{1}}{\rho}\left(\int_{0}^{1} g(s) u(s) d s+m_{2} I^{\delta} y(1)+n_{2} I^{\delta-1} y(1)\right) .
\end{aligned}
$$

It follows the definition Riemann-Liouville fractional integral that

$$
\begin{aligned}
u(t)= & -\frac{1}{\Gamma(\delta)} \int_{0}^{t}(t-s)^{\delta-1} y(s) d s \\
& +\frac{n_{1}+m_{1} t}{\rho \Gamma(\delta)} \\
& \times\left(\int_{0}^{1} m_{2}(1-s)^{\delta-1} y(s) d s\right. \\
& \left.+\int_{0}^{1} n_{2}(\delta-1)(1-s)^{\delta-2} y(s) d s\right) \\
& +\frac{n_{1}+m_{1} t}{\rho} \int_{0}^{1} g(s) u(s) d s,
\end{aligned}
$$

that is,

$$
u(t)=\int_{0}^{1} G(t, s) y(s) d s+\frac{n_{1}+m_{1} t}{\rho} \int_{0}^{1} g(s) u(s) d s .
$$

We multiply by the function $g$ on both sides of (16), integrate from 0 to 1 ,

$$
\begin{aligned}
\int_{0}^{1} g(s) u(s) d s= & \int_{0}^{1} g(s)\left(\int_{0}^{1} G(s, r) y(r) d r\right) d s \\
& +\frac{\int_{0}^{1} g(s) u(s) d s}{\rho} \int_{0}^{1}\left(n_{1}+m_{1} s\right) g(s) d s \\
= & \int_{0}^{1}\left(\int_{0}^{1} g(r) G(r, s) d r\right) y(s) d s \\
& +\frac{\int_{0}^{1} g(s) u(s) d s}{\rho} \int_{0}^{1}\left(n_{1}+m_{1} s\right) g(s) d s,
\end{aligned}
$$

and we can easily get

$$
\begin{aligned}
\int_{0}^{1} g(s) u(s) d s & \\
= & \frac{\rho}{\rho-\int_{0}^{1}\left(n_{1}+m_{1} s\right) g(s) d s} \\
& \times \int_{0}^{1}\left(\int_{0}^{1} g(r) G(r, s) d r\right) y(s) d s \\
= & \rho \bar{g} \int_{0}^{1}\left(\int_{0}^{1} g(r) G(r, s) d r\right) y(s) d s
\end{aligned}
$$

So,

$$
\begin{aligned}
u(t)= & \int_{0}^{1} G(t, s) y(s) d s+\frac{n_{1}+m_{1} t}{\rho} \\
& \cdot \rho \bar{g} \int_{0}^{1}\left(\int_{0}^{1} g(r) G(r, s) d r\right) y(s) d s \\
= & \int_{0}^{1} G(t, s) y(s) d s+\int_{0}^{1} h(t, s) y(s) d s,
\end{aligned}
$$

where $h(t, s)=\bar{g}\left(n_{1}+m_{1} t\right) \int_{0}^{1} g(r) G(r, s) d r$.

Hence,

$$
u(t)=\int_{0}^{1} H(t, s) y(s) d s .
$$

It follows from (12) that

$$
\begin{aligned}
u^{\prime}(t)= & -I^{\delta-1} y(t) \\
& +\frac{m_{1}}{\rho}\left(\int_{0}^{1} g(s) u(s) d s+m_{2} I^{\delta} y(1)+n_{2} I^{\delta-1} y(1)\right) .
\end{aligned}
$$

We can obtain $u \in A C^{2}(J)$ and ${ }^{C} D^{\delta} u(t) \in C(J)$.

Similarly, we can obtain the following lemma. 
Lemma 7. Suppose (H1) holds and $a \in \mathbb{R}$. Then the boundary value problem

$$
\begin{gathered}
-{ }^{C} D^{\delta} u(t)=0, \quad t \in J, \\
m_{1} u(0)-n_{1} u^{\prime}(0)=0, \\
m_{2} u(1)+n_{2} u^{\prime}(1)=\int_{0}^{1} g(s) u(s) d s+a
\end{gathered}
$$

has the unique solution

$$
u^{*}(t)=\bar{g} a\left(n_{1}+m_{1} t\right) .
$$

Lemma 8. Suppose (H1) holds. Then the solution $u \in A C^{2}(J)$ and ${ }^{C} D^{\delta} u(t) \in C(J)$ of the boundary value problem (1) is equivalent to the solution $u \in C(J)$ of the integral equation

$$
u(t)=\int_{0}^{1} H(t, s) f(s, u(s)) d s+\bar{g} a\left(n_{1}+m_{1} t\right), \quad t \in J .
$$

Proof. If $u \in A C^{2}(J)$ and ${ }^{C} D^{\delta} u(t) \in C(J)$ is the solution of the boundary value problem $(1)$, by Lemma $4,-{ }^{C} D^{\delta} u(t)=$ $f(t, u(t))$ is equivalent to the equation

$$
u(t)=-I^{\delta} f(t, u(t))+c_{0}+c_{1} t
$$

where $c_{0}=u(0), c_{1}=u^{\prime}(0)$.

For convenience, we denote $y(t)=f(t, u(t))$.

Similar to the proof of Lemma 6, we can obtain

$$
\begin{aligned}
& c_{0}=\frac{n_{1}}{\rho}\left(\int_{0}^{1} g(s) u(s) d s+m_{2} I^{\delta} y(1)+n_{2} I^{\delta-1} y(1)+a\right), \\
& c_{1}=\frac{m_{1}}{\rho}\left(\int_{0}^{1} g(s) u(s) d s+m_{2} I^{\delta} y(1)+n_{2} I^{\delta-1} y(1)+a\right) .
\end{aligned}
$$

Substituting $c_{0}$ and $c_{1}$ into (25), we can obtain

$$
\begin{aligned}
u(t)= & \int_{0}^{1} G(t, s) y(s) d s \\
& +\frac{n_{1}+m_{1} t}{\rho} \int_{0}^{1} g(s) u(s) d s+\frac{\left(n_{1}+m_{1} t\right) a}{\rho} .
\end{aligned}
$$

We multiply by the function $g$ on both sides of (27), integrate from 0 to 1 , and get

$$
\begin{aligned}
\int_{0}^{1} g(s) u(s) d s= & \bar{g} \rho \int_{0}^{1}\left(\int_{0}^{1} g(r) G(r, s) d r\right) y(s) d s \\
& +\bar{g} a \int_{0}^{1}\left(n_{1}+m_{1} s\right) g(s) d s .
\end{aligned}
$$

Since $\bar{g}=1 /\left(\rho-\int_{0}^{1}\left(n_{1}+m_{1} s\right) g(s) d s\right)$, we have

$$
u(t)=\int_{0}^{1} H(t, s) y(s) d s+\bar{g} a\left(n_{1}+m_{1} t\right) .
$$

That is,

$$
u(t)=\int_{0}^{1} H(t, s) f(s, u(s)) d s+\bar{g} a\left(n_{1}+m_{1} t\right) .
$$

On the other hand, if $u \in C(J)$ is the solution of the integral equation (24), we have

$$
\begin{aligned}
u^{\prime}(t)= & -\frac{1}{\Gamma(\delta-1)} \int_{0}^{t}(t-s)^{\delta-2} f(s, u(s)) d s \\
+ & \frac{m_{1}}{\rho \Gamma(\delta)} \int_{0}^{1}\left(m_{2}(1-s)^{\delta-1}\right. \\
& \left.+n_{2}(\delta-1)(1-s)^{\delta-2}\right) \\
& \times f(s, u(s)) d s \\
+ & \bar{g} m_{1} \int_{0}^{1}\left(\int_{0}^{1} g(r) G(r, s) d r\right) d s+\bar{g} a m_{1} .
\end{aligned}
$$

It is easy to see that $u^{\prime} \in A C(J)$.

Hence, $u \in A C^{2}(J)$ and ${ }^{C} D^{\delta} u \in C(J)$

We can easily verify that $u$ satisfies the boundary value problem (1).

Therefore, $u \in A C^{2}(J)$ and ${ }^{C} D^{\delta} u(t) \in C(J)$ is the solution of the boundary value problem (1).

Lemma 9. Suppose (H1) holds. Then the functions $G, h$, and $H$ have the following properties;

(1) $G \in C([0,1] \times[0,1)), 0 \leq G(t, s) \leq\left(\left(\left(m_{1}+n_{1}\right)\left(m_{2}+\right.\right.\right.$ $\left.\left.\left.n_{2}(\delta-1)\right)\right) / \Gamma(\delta) \rho\right)(1-s)^{\delta-2}$ for $(t, s) \in[0,1] \times[0,1)$ and there exists a constant $0<\gamma_{1}<1$ such that for $s \in[0,1)$

$$
\min _{t \in J} G(t, s) \geq \gamma_{1} \max _{t \in J} G(t, s) ;
$$

(2) $h(t, s) \geq 0$ for $(t, s) \in[0,1] \times[0,1)$ and there exists $a$ constant $0<\gamma_{2}<1$ such that for $s \in[0,1)$

$$
\min _{t \in J} h(t, s)=\gamma_{2} \max _{t \in J} h(t, s)
$$

(3) $H(t, s) \geq 0$ for $(t, s) \in[0,1] \times[0,1)$ and for $s \in[0,1)$

$$
\min _{t \in J} H(t, s) \geq \gamma \max _{t \in J} H(t, s),
$$

where $\gamma=\min \left\{\gamma_{1}, \gamma_{2}\right\}$.

Proof. (1) By the expression of $G(t, s)$, it is easy to see $G \in$ $C([0,1] \times[0,1))$.

Since $(\mathrm{H} 1)$ holds, we can show that for $(t, s) \in[0,1] \times[0,1)$

$$
G(t, s) \leq \frac{\left(n_{1}+m_{1}\right)\left(m_{2}+n_{2}(\delta-1)\right)}{\rho \Gamma(\delta)}(1-s)^{\delta-2} .
$$

For $0 \leq t \leq s<1$, it follows

$$
\begin{aligned}
G(t, s) & \geq \frac{n_{1} n_{2}(\delta-1)(1-s)^{\delta-2}}{\rho \Gamma(\delta)} \\
& \geq \frac{n_{2}(1-s)^{\delta-2}}{\rho \Gamma(\delta)}\left(n_{1}(\delta-1)-(2-\delta) m_{1}\right) .
\end{aligned}
$$


For $0 \leq s<t \leq 1$, by (10), we have

$$
\begin{gathered}
\frac{\partial G(t, s)}{\partial t}=\frac{1}{\rho \Gamma(\delta)}\left(m _ { 1 } \left(m_{2}(1-s)^{\delta-1}\right.\right. \\
\left.+n_{2}(\delta-1)(1-s)^{\delta-2}\right) \\
\left.-\rho(\delta-1)(t-s)^{\delta-2}\right), \\
\frac{\partial^{2} G(t, s)}{\partial t^{2}}=\frac{1}{\rho \Gamma(\delta)}\left(-\rho(\delta-1)(\delta-2)(t-s)^{\delta-3}\right)>0 .
\end{gathered}
$$

It is easy to see that

$$
\begin{aligned}
\max _{t \in[s, 1]} \frac{\partial G(t, s)}{\partial t} & \\
= & \left.\frac{\partial G(t, s)}{\partial t}\right|_{t=1} \\
= & \frac{1}{\rho \Gamma(\delta)}\left(m_{1}\left(m_{2}(1-s)^{\delta-1}+n_{2}(\delta-1)(1-s)^{\delta-2}\right)\right. \\
& \left.-\rho(\delta-1)(1-s)^{\delta-2}\right) \\
= & \frac{m_{2}(1-s)^{\delta-2}}{\rho \Gamma(\delta)}\left(m_{1}(1-s)-\left(m_{1}+n_{1}\right)(\delta-1)\right) .
\end{aligned}
$$

(H1) implies that $\left(m_{1}+n_{1}\right)(\delta-1)>m_{1}$. By (38), we have $\max _{t \in[s, 1]}(\partial G(t, s) / \partial t) \leq 0$. Hence,

$$
\begin{aligned}
\min _{t \in[s, 1]} G(t, s) & =G(1, s) \\
& =\frac{n_{2}(1-s)^{\delta-2}}{\rho \Gamma(\delta)}\left(\left(n_{1}+m_{1}\right)(\delta-1)-m_{1}(1-s)\right) \\
& \geq \frac{n_{2}(1-s)^{\delta-2}}{\rho \Gamma(\delta)}\left(\left(n_{1}+m_{1}\right)(\delta-1)-m_{1}\right)
\end{aligned}
$$

For $(t, s) \in[0,1] \times[0,1)$, we can show that

$$
G(t, s) \geq \frac{n_{2}(1-s)^{\delta-2}}{\rho \Gamma(\delta)}\left(\left(n_{1}+m_{1}\right)(\delta-1)-m_{1}\right)>0
$$

from (36) and (39).

We denote $\gamma_{1}=n_{2}\left(\left(n_{1}+m_{1}\right)(\delta-1)-m_{1}\right) /\left(n_{1}+m_{1}\right)\left(m_{2}+\right.$ $\left.n_{2}(\delta-1)\right)$. It is obvious that $0<\gamma_{1}<1$.

By (35) and (39), for $(t, s) \in[0,1] \times[0,1)$, we have

$$
\begin{aligned}
\min _{t \in J} G(t, s) \geq & \frac{n_{2}\left(\left(n_{1}+m_{1}\right)(\delta-1)-m_{1}\right)}{\rho \Gamma(\delta)}(1-s)^{\delta-2} \\
\geq & \frac{n_{2}\left(\left(n_{1}+m_{1}\right)(\delta-1)-m_{1}\right)}{\rho \Gamma(\delta)} \\
& \cdot \frac{\rho \Gamma(\delta)}{\left(n_{1}+m_{1}\right)\left(m_{2}+n_{2}(\delta-1)\right)} \\
& \cdot G(t, s)=\gamma_{1} G(t, s) .
\end{aligned}
$$

Hence, $\min _{t \in J} G(t, s) \geq \gamma_{1} \max _{t \in J} G(t, s)$.

(2) By the expression of $h(t, s)$, see (11), it is easy to see $h(t, s) \geq 0$ for $(t, s) \in[0,1] \times[0,1)$ and

$$
\min _{t \in J} h(t, s)=\gamma_{2} \max _{t \in J} h(t, s) \quad \text { for } s \in[0,1),
$$

where $\gamma_{2}=n_{1} /\left(n_{1}+m_{1}\right)$.

(3) By (1) and (2), we have $H(t, s) \geq 0$ for $(t, s) \in[0,1] \times$ $[0,1)$ and

$$
\begin{aligned}
\min _{t \in J} H(t, s) & =\min _{t \in J}(G(t, s)+h(t, s)) \\
& \geq \min _{t \in J} G(t, s)+\min _{t \in J} h(t, s) \\
& \geq \gamma_{1} \max _{t \in J} G(t, s)+\gamma_{2} \max _{t \in J} h(t, s) \\
& \geq \gamma\left(\max _{t \in J} G(t, s)+\max _{t \in J} h(t, s)\right) \\
& \geq \gamma \max _{t \in J} H(t, s) .
\end{aligned}
$$

For the sake of the reader, we state the fixed point index theorem and Schauder's fixed point theorem which will be used later.

Lemma 10 (See [38]). Let $P$ be a cone of a real Banach space $E, \Omega$ be a bounded open set in $E$, and $\theta \in \Omega$. Suppose $A$ : $P \cap \bar{\Omega} \rightarrow P$ is a completely continuous operator. If $A u \neq \rho u$ for any $u \in \partial \Omega \cap P$ and $\rho \geq 1$, then

$$
i(A, \Omega \cap P, P)=1 \text {. }
$$

Lemma 11 (Schauder's fixed point theorem, see [39]). Let $E$ be a real Banach space, and let $\Omega \subset E$ be nonempty closed bounded and convex; $A: \Omega \rightarrow \Omega$ compact. Then $A$ has a fixed point.

\section{Comparison Principle and the Existence of Solutions}

Definition 12. Let $\alpha \in A C^{2}(J)$ and ${ }^{C} D^{\delta} \alpha \in C(J)$. One says that $\alpha$ is a lower solution of the boundary value problem (1), if

$$
\begin{gathered}
-{ }^{C} D^{\delta} \alpha(t) \leq f(t, \alpha(t))+p_{\alpha}(t), \quad t \in J, \\
m_{1} \alpha(0)-n_{1} \alpha^{\prime}(0)=0,
\end{gathered}
$$

where

$$
p_{\alpha}(t)=\left\{\begin{array}{r}
0, \\
m_{2} \alpha(1)+n_{2} \alpha^{\prime}(1) \leq \int_{0}^{1} g(s) \alpha(s) d s+a, \\
\left({ }^{C} D^{\delta} c(t)\right)\left(\int_{0}^{1} g(s) \alpha(s) d s\right. \\
\left.+a-m_{2} \alpha(1)-n_{2} \alpha^{\prime}(1)\right) \\
m_{2} \alpha(1)+n_{2} \alpha^{\prime}(1)>\int_{0}^{1} g(s) \alpha(s) d s+a .
\end{array}\right.
$$


Let $\beta \in A C^{2}(J)$ and ${ }^{C} D^{\delta} \beta \in C(J)$. We say that $\beta$ is an upper solution of the boundary value problem (1), if

$$
\begin{gathered}
-{ }^{C} D^{\delta} \beta(t) \geq f(t, \beta(t))+q_{\beta}(t), \quad t \in J, \\
m_{1} \beta(0)-n_{1} \beta^{\prime}(0)=0,
\end{gathered}
$$

where

$$
q_{\beta}(t)=\left\{\begin{aligned}
& 0, \\
& m_{2} \beta(1)+n_{2} \beta^{\prime}(1) \geq \int_{0}^{1} g(s) \beta(s) d s+a, \\
&\left({ }^{C} D^{\delta} c(t)\right)\left(\int_{0}^{1} g(s) \beta(s) d s\right. \\
&\left.+a-m_{2} \beta(1)-n_{2} \beta^{\prime}(1)\right), \\
& m_{2} \beta(1)+n_{2} \beta^{\prime}(1)<\int_{0}^{1} g(s) \beta(s) d s+a,
\end{aligned}\right.
$$

where $c(t)$ is defined in (H2).

The following comparison principle will play a very important role in our main results analysis.

Lemma 13. Let (H1) hold. Suppose that $u \in A C^{2}(J),{ }^{C} D^{\delta} u \in$ $C(J)$, and satisfies

$$
\begin{gathered}
{ }^{C} D^{\delta} u(t) \geq 0, \\
m_{1} u(0)-n_{1} u^{\prime}(0)=0, \\
m_{2} u(1)+n_{2} u^{\prime}(1) \geq \int_{0}^{1} g(s) u(s) d s .
\end{gathered}
$$

Then $u(t) \geq 0$ for $t \in J$.

Proof. Denote

$$
-{ }^{C} D^{\delta} u(t)=y(t),
$$

then $y(t) \geq 0$ for $t \in J$. Let $a \geq 0$ such that

$$
m_{2} u(1)+n_{2} u^{\prime}(1)=\int_{0}^{1} g(s) u(s) d s+a .
$$

By Lemma 8, we can get that the boundary value problem

$$
\begin{gathered}
{ }^{C} D^{\delta} u(t)=y(t), \quad t \in J, \\
m_{1} u(0)-n_{1} u^{\prime}(0)=0, \\
m_{2} u(1)+n_{2} u^{\prime}(1)=\int_{0}^{1} g(s) u(s) d s+a
\end{gathered}
$$

has unique solution

$$
u(t)=\int_{0}^{1} H(t, s) y(s) d s+u^{*}(t)
$$

where $u^{*}(t)=\bar{g} a\left(n_{1}+m_{1} t\right)$ and $t \in J$.

It follows that $u(t) \geq 0$ for $t \in J$ from Lemma 9 .
Lemma 14. Let (H1) and (H2) hold. Suppose that $u \in A C^{2}(J)$, ${ }^{C} D^{\delta} u \in C(J)$, and satisfies

$$
\begin{aligned}
& -{ }^{C} D^{\delta} u(t)-\left({ }^{C} D^{\delta} c(t)\right) \\
& \quad \times\left(\int_{0}^{1} g(s) u(s) d s-m_{2} u(1)-n_{2} u^{\prime}(1)\right) \geq 0, \\
& m_{1} u(0)-n_{1} u^{\prime}(0)=0, \\
& m_{2} u(1)+n_{2} u^{\prime}(1)<\int_{0}^{1} g(s) u(s) d s . \\
& \text { Then } u(t) \geq 0 \text { for } t \in J .
\end{aligned}
$$

Proof. Let

$$
\begin{aligned}
v(t)=u(t)+c(t)\left(\int_{0}^{1} g(s) u(s) d s\right. & \\
& \left.-m_{2} u(1)-n_{2} u^{\prime}(1)\right) .
\end{aligned}
$$

Since (H2) holds, we have $c(t) \leq 0$ for $t \in J, m_{1} c(0)-$ $n_{1} c^{\prime}(0)=0, m_{2} c(1)+n_{2} c^{\prime}(1) \geq-1$, and $\int_{0}^{1} g(s) c(s) d s \leq-2$.

Hence, by (54), we have

$$
u(t) \geq v(t), \quad \text { for } t \in J,
$$

$$
\begin{aligned}
-{ }^{C} D^{\delta} v(t) \\
=-{ }^{C} D^{\delta} u(t)-\left({ }^{C} D^{\delta} c(t)\right) \\
\quad \times\left(\int_{0}^{1} g(s) u(s) d s-m_{2} u(1)-n_{2} u^{\prime}(1)\right) \geq 0, \\
m_{1} v(0)-n_{1} v^{\prime}(0) \\
=m_{1} u(0)-n_{1} u^{\prime}(0) \\
\quad+\left(\int_{0}^{1} g(s) u(s) d s-m_{2} u(1)-n_{2} u^{\prime}(1)\right) \\
\quad \times\left(m_{1} c(0)-n_{1} c^{\prime}(0)\right) \\
=m_{1} u(0)-n_{1} u^{\prime}(0)=0, \\
m_{2} v(1)+n_{2} v^{\prime}(1) \\
=m_{2} u(1)+n_{2} u^{\prime}(1) \\
\quad+\left(\int_{0}^{1} g(s) u(s) d s-m_{2} u(1)-n_{2} u^{\prime}(1)\right) \\
\quad \times\left(m_{2} c(1)+n_{2} c^{\prime}(1)\right)
\end{aligned}
$$




$$
\begin{aligned}
\geq & m_{2} u(1)+n_{2} u^{\prime}(1) \\
& -\left(\int_{0}^{1} g(s) u(s) d s-m_{2} u(1)-n_{2} u^{\prime}(1)\right) \\
= & (-2)\left(\int_{0}^{1} g(s) u(s) d s-m_{2} u(1)-n_{2} u^{\prime}(1)\right) \\
& +\int_{0}^{1} g(s) u(s) d s \\
\geq & \int_{0}^{1} g(s) c(s) d s \\
& \times\left(\int_{0}^{1} g(s) u(s) d s-m_{2} u(1)-n_{2} u^{\prime}(1)\right) \\
& +\int_{0}^{1} g(s) u(s) d s \\
= & \int_{0}^{1} g(s) v(s) d s .
\end{aligned}
$$

In view of Lemma $13, v(t) \geq 0$ for $t \in J$, which implies that $u(t) \geq 0$.

We can easily obtain the following lemma from the definition of $u^{*}$, where $u^{*}$ is defined by Lemma 7 .

Lemma 15. Suppose (H1) holds. $u$ is a positive solution of the boundary value problem (1) if and only if $w=u-u^{*}$ is a positive solution of the boundary value problem

$$
\begin{gathered}
-{ }^{C} D^{\delta} w(t)=f\left(t, w(t)+u^{*}(t)\right), \quad t \in J, \\
m_{1} w(0)-n_{1} w^{\prime}(0)=0, \\
m_{2} w(1)+n_{2} w^{\prime}(1)=\int_{0}^{1} g(s) w(s) d s .
\end{gathered}
$$

Let $E=C(J)$ be the Banach space with the norm $\|u\|=$ $\max _{t \in J}|u(t)|$,

$$
P=\{u \in E: u(t) \geq 0 \text { for } t \in J\},
$$

and let

$$
P_{0}=\{u \in E: u(t) \geq 0, u(t) \geq \gamma\|u\| \text { for } t \in J\}
$$

be cones in $E$, and $P_{0} \subset P$, where $\gamma$ is defined by Lemma 9 .

Lemma 16. Suppose (H1) holds. If $w$ is a positive solution of the boundary value problem (57), then

$$
\min _{t \in J} w(t) \geq \gamma\|w\|
$$

Proof. By Lemma 6, we can show that the solution $w$ of the boundary value problem (57) satisfies

$$
w(t)=\int_{0}^{1} H(t, s) f\left(s, w(s)+u^{*}(s)\right) d s .
$$

In view of Lemma 9(3),

$$
\begin{aligned}
\min _{t \in J} w(t) & =\min _{t \in J} \int_{0}^{1} H(t, s) f\left(s, w(s)+u^{*}(s)\right) d s \\
& \geq \int_{0}^{1} \gamma \max _{t \in J} H(t, s) f\left(s, w(s)+u^{*}(s)\right) d s \\
& \geq \gamma \max _{t \in J} \int_{0}^{1} H(t, s) f\left(s, w(s)+u^{*}(s)\right) d s \\
& =\gamma \max _{t \in J} w(t)=\gamma\|w\| .
\end{aligned}
$$

We define $T: P \rightarrow E$ by

$$
T w(t)=\int_{0}^{1} H(t, s) f\left(s, w(s)+u^{*}(s)\right) d s .
$$

By Lemma 16, we have $T: P \rightarrow P_{0}$. Since $f$ and $H$ are nonnegative, then $u$ is a positive solution of (1) if and only if $w \in P$ is a fixed point of the operator $T$.

Theorem 17. Suppose (H1), (H2), and (H3) hold and there exist a nonnegative lower solution $\alpha$ and an upper solution $\beta$ of the boundary value problem (1) such that $\alpha \leq \beta$. Then the boundary value problem (1) has at least one solution $u$ such that $\alpha \leq u \leq \beta$.

Proof. Let

$$
F(t, u)= \begin{cases}f(t, \beta(t)), & u>\beta(t) \\ f(t, u), & \alpha(t) \leq u \leq \beta(t), \\ f(t, \alpha(t)), & u<\alpha(t)\end{cases}
$$

We consider the boundary value problem

$$
\begin{gathered}
-{ }^{C} D^{\delta} u(t)=F(t, u(t)), \quad t \in J, \\
m_{1} u(0)-n_{1} u^{\prime}(0)=0, \\
m_{2} u(1)+n_{2} u^{\prime}(1)=\int_{0}^{1} g(s) u(s) d s+a .
\end{gathered}
$$

By Lemma $15, u$ is a positive solution of the boundary value problem (65) if and only if $w=u-u^{*}$ is a positive solution of the following boundary value problem:

$$
\begin{gathered}
-{ }^{C} D^{\delta} w(t)=F\left(t, w(t)+u^{*}(t)\right), \quad t \in J, \\
m_{1} w(0)-n_{1} w^{\prime}(0)=0, \\
m_{2} w(1)+n_{2} w^{\prime}(1)=\int_{0}^{1} g(s) w(s) d s .
\end{gathered}
$$

We define $\widehat{T}: P \rightarrow E$ by

$$
\widehat{T} w(t)=\int_{0}^{1} H(t, s) F\left(s, w(s)+u^{*}(s)\right) d s .
$$


Then $w$ is a solution of (66) if and only if $w$ is a fixed point of the operator $\widehat{T}$. It is easy to see $\widehat{T}: P \rightarrow P_{0}$.

Next we can prove that $\widehat{T}$ is completely continuous.

Let $D \subset P$ be a bounded set, and there exists a constant $M_{0}>0$ such that $\|w\| \leq M_{0}$ for $w \in D$. Because $F$ is continuous, there exists a constant $M_{1}>0$ such that $\max _{s \in J, w \in D}\left|F\left(s, w(s)+u^{*}(s)\right)\right| \leq M_{1}$. We have

$$
|\widehat{T} w(t)| \leq M_{1} \int_{0}^{1} H(t, s) d s \leq M_{1} \max _{t \in J} \int_{0}^{1} H(t, s) d s .
$$

So $\widehat{T}(D)$ is uniformly bounded.

We denote

$$
\begin{aligned}
G_{1}(t, s)= & \frac{1}{\rho \Gamma(\delta)}\left(n_{1}+m_{1} t\right) \\
& \times\left(m_{2}(1-s)^{\delta-1}+n_{2}(\delta-1)(1-s)^{\delta-2}\right), \\
G_{2}(t, s) & =\frac{1}{\Gamma(\delta)} \begin{cases}-(t-s)^{\delta-1}, & 0 \leq s \leq t \leq 1, \\
0, & 0 \leq t<s \leq 1,\end{cases} \\
z(s)= & \frac{1}{\rho \Gamma(\delta)} \\
& \times\left(m_{2}(1-s)^{\delta-1}+n_{2}(\delta-1)(1-s)^{\delta-2}\right) \\
& +\bar{g} \int_{0}^{1} g(r) G(r, s) d r .
\end{aligned}
$$

Hence,

$$
\begin{aligned}
H(t, s) & =G(t, s)+h(t, s) \\
& =G_{1}(t, s)+G_{2}(t, s)+h(t, s) \\
& =\left(n_{1}+m_{1} t\right) z(s)+G_{2}(t, s),
\end{aligned}
$$

where $h(t, s)$ is defined by (11).

Since $G_{2}(t, s)$ is continuous on $J \times J$, we have $G_{2}$ which is uniformly continuous on $J \times J$. It implies that for any $\varepsilon>0$, there exists $\delta_{1}>0$, when $t_{1}, t_{2} \in J$; whenever $\left|t_{2}-t_{1}\right|<\delta_{1}$ and $s \in J$, we can obtain

$$
\left|G_{2}\left(t_{2}, s\right)-G_{2}\left(t_{1}, s\right)\right|<\frac{\varepsilon}{2 M_{1}} .
$$

By (H1), it is easy to see $\int_{0}^{1} z(s) d s \neq 0$. We take $0<\delta_{0} \leq$ $\min \left\{\delta_{1}, \varepsilon /\left(2 M_{1}\left(m_{1}+1\right) \int_{0}^{1} z(s) d s\right)\right\}$.
Therefore, as $t_{1}, t_{2} \in J$, whenever $\left|t_{2}-t_{1}\right|<\delta_{0}$ and $u \in D$, we can show that

$$
\begin{aligned}
& \left|\widehat{T} w\left(t_{2}\right)-\widehat{T} w\left(t_{1}\right)\right| \\
& =\left|\int_{0}^{1}\left(H\left(t_{2}, s\right)-H\left(t_{1}, s\right)\right) F\left(s, w(s)+u^{*}(s)\right) d s\right| \\
& \leq M_{1} \int_{0}^{1}\left|H\left(t_{2}, s\right)-H\left(t_{1}, s\right)\right| d s \\
& \leq M_{1} \int_{0}^{1} m_{1}\left|t_{2}-t_{1}\right| z(s) d s \\
& \quad+M_{1} \int_{0}^{1}\left|G_{2}\left(t_{2}, s\right)-G_{2}\left(t_{1}, s\right)\right| d s<\varepsilon .
\end{aligned}
$$

Thus, we have proved $\widehat{T}$ is equicontinuous.

By Arzela-Ascoli theorem, we know that $\widehat{T}(D)$ is relatively compact.

We can easily show that $\widehat{T}$ is continuous since $F$ is continuous. Hence, $\widehat{T}$ is completely continuous.

Since $F$ is bounded and $\widehat{T}$ is completely continuous, we can get $\widehat{T}$ has at least one fixed point $w$ by Schauder fixed point theorem, that is, there exists a solution $w$ of the boundary value problem (66).

Then $u=w+u^{*}$ is a solution of the boundary value problem (65).

Finally, we prove $\alpha(t) \leq u(t) \leq \beta(t)$, for $t \in J$.

We can prove that if each solution $u$ of the boundary value problem (65) satisfies $\alpha(t) \leq u(t) \leq \beta(t)$ for $t \in J$, then $u$ is a solution of the boundary value problem (1).

Let $v(t)=u(t)-\alpha(t)$, for $t \in J$.

If $m_{2} \alpha(1)+n_{2} \alpha^{\prime}(1) \leq \int_{0}^{1} g(s) \alpha(s) d s+a$, since $f(t, u)$ is monotonically increasing with respect to $u$ and $\alpha$ is a lower solution of (1), we have

$$
\begin{gathered}
{ }_{-} D^{\delta} v(t)=-{ }^{C} D^{\delta} u(t)+{ }^{C} D^{\delta} \alpha(t) \\
\geq F(t, u(t))-f(t, \alpha(t)) \geq 0, \\
m_{1} v(0)-n_{1} v^{\prime}(0) \\
=m_{1} u(0)-n_{1} u^{\prime}(0)-\left(m_{1} \alpha(0)-n_{1} \alpha^{\prime}(0)\right)=0, \\
m_{2} v(1)+n_{2} v^{\prime}(1) \\
=\left(m_{2} u(1)+n_{2} u^{\prime}(1)\right)-\left(m_{2} \alpha(1)+n_{2} \alpha^{\prime}(1)\right), \\
\geq\left(\int_{0}^{1} g(s) u(s) d s+a\right)-\left(\int_{0}^{1} g(s) \alpha(s) d s+a\right) \\
=\int_{0}^{1} g(s) v(s) d s .
\end{gathered}
$$

It follows that $v(t) \geq 0$ on $J$ from Lemma 13 . 


$$
\begin{aligned}
& \text { If } m_{2} \alpha(1)+n_{2} \alpha^{\prime}(1)>\int_{0}^{1} g(s) \alpha(s) d s+a \text {, we have } \\
& -{ }^{C} D^{\delta} v(t)-\left({ }^{C} D^{\delta} c(t)\right) \\
& \quad \times\left(\int_{0}^{1} g(s) v(s) d s-m_{2} v(1)-n_{2} v^{\prime}(1)\right) \\
& =-{ }^{C} D^{\delta} u(t)-\left({ }^{C} D^{\delta} c(t)\right) \\
& \quad \times\left(\int_{0}^{1} g(s) u(s) d s-m_{2} u(1)-n_{2} u^{\prime}(1)\right) \\
& -\left(-{ }^{C} D^{\delta} \alpha(t)-\left({ }^{C} D^{\delta} c(t)\right)\right. \\
& \left.\quad \times\left(\int_{0}^{1} g(s) \alpha(s) d s-m_{2} \alpha(1)-n_{2} \alpha^{\prime}(1)\right)\right) \\
& \geq F(t, u(t))-\left({ }^{C} D^{\delta} c(t)\right) \\
& \quad \times\left(\int_{0}^{1} g(s) u(s) d s-m_{2} u(1)-n_{2} u^{\prime}(1)\right) \\
& -\left(f(t, \alpha(t))+\left({ }^{C} D^{\delta} c(t)\right)\right. \\
& \quad \times\left(\int_{0}^{1} g(s) \alpha(s) d s+a-m_{2} \alpha(1)-n_{2} \alpha^{\prime}(1)\right) \\
& \quad-\left({ }^{C} D^{\delta} c(t)\right)\left(\int_{0}^{1} g(s) \alpha(s) d s\right. \\
& \left.\left.\quad-m_{2} \alpha(1)-n_{2} \alpha^{\prime}(1)\right)\right)
\end{aligned}
$$

By (H3), we can get $F(t, u(t))-f(t, \alpha(t)) \geq 0$, that is,

$$
\begin{aligned}
& -{ }^{C} D^{\delta} v(t)-\left({ }^{C} D^{\delta} c(t)\right) \\
& \quad \times\left(\int_{0}^{1} g(s) v(s) d s-m_{2} v(1)-n_{2} v^{\prime}(1)\right) \geq 0 .
\end{aligned}
$$

So,

$$
\begin{aligned}
& m_{1} v(0)-n_{1} v^{\prime}(0) \\
& =\left(m_{1} u(0)-n_{1} u^{\prime}(0)\right)-\left(m_{1} \alpha(0)-n_{1} \alpha^{\prime}(0)\right)=0, \\
& m_{2} v(1)+n_{2} v^{\prime}(1) \\
& \quad=m_{2} u(1)+n_{2} u^{\prime}(1)-\left(m_{2} \alpha(1)+n_{2} \alpha^{\prime}(1)\right) \\
& \quad<\int_{0}^{1} g(s) u(s) d s+a-\left(\int_{0}^{1} g(s) \alpha(s) d s+a\right) \\
& \quad=\int_{0}^{1} g(s) v(s) d s .
\end{aligned}
$$

It follows that $v(t) \geq 0$ on $J$ from Lemma 14 .
Hence, we show $\alpha(t) \leq u(t)$ on $J$.

Similarly, we can get $u(t) \leq \beta(t)$ on $J$.

Therefore, each solution $u$ of the boundary value problem (65) satisfies $\alpha(t) \leq u(t) \leq \beta(t)$ for $t \in J$. That is, $F(t, u(t))=$ $f(t, u(t))$, and $u$ is a solution of the boundary value problem $(1)$.

Theorem 18. Suppose (H1), (H2), and (H3) hold:

(1) if there exists a constant $\bar{a}>0$ such that the boundary value problem

$$
\begin{gathered}
-{ }^{C} D^{\delta} u(t)=f(t, u(t)), \quad t \in J, \\
m_{1} u(0)-n_{1} u^{\prime}(0)=0, \\
m_{2} u(1)+n_{2} u^{\prime}(1)=\int_{0}^{1} g(s) u(s) d s+\bar{a}
\end{gathered}
$$

has a positive solution $\bar{u}(t)$, then for each a with $0 \leq a \leq \bar{a}$, the boundary value problem (1) has a positive solution $u$ and $u^{*} \leq u \leq \bar{u}$, where $u^{*}(t)=\bar{g} a\left(n_{1}+m_{1} t\right)$ for $t \in J$;

(2) if there exists a constant $\bar{a}>0$ such that the boundary value problem (77) does not have positive solutions, then for each $a>\bar{a}$, the boundary value problem (1) does not have positive solutions.

Proof. (1) Let $\bar{u}^{*}(t)=\overline{g a}\left(n_{1}+m_{1} t\right)$ for $t \in J$. By Lemma 15 , $w=\bar{u}-\bar{u}^{*}$ is a positive solution of the boundary value problem

$$
\begin{gathered}
-{ }^{C} D^{\delta} w(t)=f\left(t, w(t)+\bar{u}^{*}(t)\right), \quad t \in J, \\
m_{1} w(0)-n_{1} w^{\prime}(0)=0, \\
m_{2} w(1)+n_{2} w^{\prime}(1)=\int_{0}^{1} g(s) w(s) d s,
\end{gathered}
$$

which implies $\bar{u}^{*} \leq \bar{u}$.

Since $\bar{u}^{*}(t)=\bar{g} a\left(n_{1}+m_{1} t\right)>\bar{g} a\left(n_{1}+m_{1} t\right)=u^{*}(t)$, we take $\alpha=u^{*}$ and $\beta=\bar{u}$.

We can easily verify that $\alpha$ and $\beta$ are a lower solution and an upper solution of the boundary value problem (1) and $\alpha \leq$ $\beta$.

By Theorem 17, we have that the boundary value problem (1) has a positive solution $u$ and $u^{*} \leq u \leq \bar{u}$.

(2) If there exists a constant $a_{0}>\bar{a}$ such that the boundary value problem (77) has a positive solution, by (1), we can show that for each $a$ with $0 \leq a \leq a_{0}$, the boundary value problem (1) has a positive solution. So, the boundary value problem (1) has a positive solution, for $a=\bar{a}$, which is a contradiction.

Therefore, if there exists a constant $\bar{a}>0$ such that the boundary value problem (77) does not have positive solutions, then for each $a>\bar{a}$, the boundary value problem (1) does not have positive solutions. 


\section{Impact of Disturbance Parameter $a$ on the Existence of Solutions}

For convenience, we give the following notations:

$$
\begin{aligned}
f^{\infty} & =\limsup _{u \rightarrow+\infty} \max _{t \in J} \frac{f(t, u)}{u}, & f_{\infty}=\liminf _{u \rightarrow+\infty} \min _{t \in J} \frac{f(t, u)}{u}, \\
f^{0} & =\limsup _{u \rightarrow 0} \max _{t \in J} \frac{f(t, u)}{u}, & f_{0}=\liminf _{u \rightarrow 0} \min _{t \in J} \frac{f(t, u)}{u} .
\end{aligned}
$$

We can see that $\int_{0}^{1} \max _{t \in J} H(t, s) d s \neq 0$ from (H1) and we denote $\eta=1 /\left(\int_{0}^{1} \max _{t \in J} H(t, s) d s\right)$.

Lemma 19. Suppose that (H1) holds, $f_{\infty}>\eta / \gamma^{2}$, and $\Omega \subseteq$ $[0,+\infty)$ is a bounded set. Then for each $a \in \Omega$, there exists a constant $\tau$ such that the solution $u$ of the boundary value problem (1) satisfies $\|u\|<\tau$.

Proof. Since $\Omega \subseteq[0,+\infty)$ is a bounded set, there exists a constant $\sigma$ such that each $a \in \Omega$, and we have $0 \leq a \leq \sigma$.

Since $f_{\infty}>\eta / \gamma^{2}$, there exists a constant $r>0$ such that

$$
f(t, u)>\frac{\eta}{\gamma^{2}} u
$$

for any $t \in J$, and $u \geq r$.

By Lemma $15, u$ is a positive solution of the boundary value problem (1) if and only if $w=u-u^{*}$ is a positive solution of the boundary value problem (57).

In view of Lemma $16, \min _{t \in J} w(t) \geq \gamma\|w\|$. We can get $\|w\| \leq r / \gamma$. Otherwise, if $\|w\|>r / \gamma$, we have

$$
\left|w(t)+u^{*}(t)\right| \geq|w(t)| \geq \gamma\|w\| \geq r
$$

for $t \in J$. Hence,

$$
\begin{aligned}
w(t) & =\int_{0}^{1} H(t, s) f\left(s, w(s)+u^{*}(s)\right) d s \\
& >\frac{\eta}{\gamma^{2}} \int_{0}^{1} H(t, s)\left|w(s)+u^{*}(s)\right| d s \\
& \geq \frac{\eta}{\gamma^{2}} \cdot \gamma^{2}\|w\| \int_{0}^{1} \max _{t \in J} H(t, s) d s \\
& =\|w\|,
\end{aligned}
$$

which is a contradiction.

We take $\tau=(r / \gamma)+\bar{g} \sigma\left(n_{1}+m_{1}\right)+1$, then

$$
\|u\| \leq\|w\|+\left\|u^{*}\right\| \leq \frac{r}{\gamma}+\bar{g} \sigma\left(n_{1}+m_{1}\right)<\tau .
$$

Theorem 20. Suppose (H1) holds. If one of the following conditions is satisfied, then the boundary value problem (1) does not have positive solutions:
(1) $f_{0}>\eta / \gamma^{2}, a \geq 0$ and $a$ is small enough;

(2) $f_{\infty}>\eta / \gamma^{2}, a>0$ and $a$ is large enough;

(3) there exist constants $K_{1} \geq 0$ and $K_{2} \geq \eta / \gamma^{2}$ such that

$$
f(t, u)>K_{1}+K_{2} u
$$

for $(t, u) \in J \times[0,+\infty)$, and $a \geq 0$.

Proof. (1) If there exists $a \geq 0$ and $a$ is small enough such that the boundary value problem (1) has a positive solution $u$, we have that $w=u-u^{*}$ is a positive solution of the boundary value problem (57) by Lemma 15 , and $\min _{t \in J} w(t) \geq \gamma\|w\|$ by Lemma 16.

Because $\liminf \operatorname{in}_{u \rightarrow 0} \min _{t \in J}(f(t, u) / u)=f_{0}$, there exists a constant $r_{1}>0$ such that

$$
f(t, u)>\frac{\eta u}{\gamma^{2}}
$$

for any $t \in J$, and $0<u \leq r_{1}$.

We take $a \geq 0$ and $a$ is small enough such that

$$
0<\left|w(t)+u^{*}(t)\right|=w(t)+\bar{g} a\left(n_{1}+m_{1} t\right) \leq r_{1}
$$

for $t \in J$. Hence,

$$
\begin{aligned}
w(t) & =\int_{0}^{1} H(t, s) f\left(s, w(s)+u^{*}(s)\right) d s \\
& >\frac{\eta}{\gamma^{2}} \cdot \gamma \int_{0}^{1} \max _{t \in J} H(t, s)\left(w(s)+u^{*}(s)\right) d s \\
& \geq \frac{\eta}{\gamma^{2}} \cdot \gamma^{2}\|w\| \int_{0}^{1} \max _{t \in J} H(t, s) d s \\
& =\|w\|,
\end{aligned}
$$

which is a contradiction.

(2) If there exists a constant $\widehat{a}>0$ and $\hat{a}$ is large enough such that the boundary value problem (1) has a positive solution $\widehat{u}$, we have that $\widehat{w}=\widehat{u}-\widehat{u}^{*}$ is a positive solution by Lemma 15.

By liminf $\operatorname{lit}_{u \rightarrow+\infty} \min _{t \in J}(f(t, u) / u)=f_{\infty}$, there exists a constant $r_{2}>0$ such that

$$
f(t, u)>\frac{\eta u}{\gamma^{2}}
$$

for any $t \in J$, and $u \geq r_{2}$. We take $\widehat{a}>r_{2} / \bar{g} n_{1}$, then

$$
\left|\widehat{w}(t)+\widehat{u}^{*}(t)\right| \geq\left|\widehat{u}^{*}(t)\right|=\bar{g} \widehat{a}\left(n_{1}+m_{1} t\right) \geq r_{2}
$$

for $t \in J$. Hence,

$$
\begin{aligned}
\widehat{w}(t) & =\int_{0}^{1} H(t, s) f\left(s, \widehat{w}(s)+\widehat{u}^{*}(s)\right) d s \\
& >\frac{\eta}{\gamma^{2}} \cdot \gamma \int_{0}^{1} \max _{t \in J} H(t, s)\left(\widehat{w}(s)+\widehat{u}^{*}(s)\right) d s \\
& \geq \frac{\eta}{\gamma^{2}} \cdot \gamma^{2}\|\widehat{w}\| \int_{0}^{1} \max _{t \in J} H(t, s) d s \\
& =\|\widehat{w}\|,
\end{aligned}
$$

which is a contradiction. 

that

(3) Since there exist constants $K_{1} \geq 0$ and $K_{2} \geq \eta / \gamma^{2}$ such

$$
f(t, u)>K_{1}+K_{2} u
$$

for $(t, u) \in J \times[0,+\infty)$, we have

$$
\begin{aligned}
w(t) & =\int_{0}^{1} H(t, s) f\left(s, w(s)+u^{*}(s)\right) d s \\
& >\gamma \int_{0}^{1} \max _{t \in J} H(t, s)\left(K_{1}+K_{2}\left(w(s)+u^{*}(s)\right)\right) d s \\
& \geq K_{2} \gamma^{2}\|w\| \int_{0}^{1} \max _{t \in J} H(t, s) d s \\
& \geq\|w\|,
\end{aligned}
$$

which is a contradiction.

Theorem 21. Suppose (H1) holds.

(1) If $0 \leq f^{0}<\eta$, then the boundary value problem (1) has at least one positive solution when $a \geq 0$ and $a$ is small enough.

(2) If $0 \leq f^{\infty}<\eta$, then the boundary value problem (1) has at least one positive solution when $a \geq 0$.

Proof. (1) Because limsup $\sup _{u \rightarrow 0} \max _{t \in J}(f(t, u) / u)=f^{0}$, there exists a constant $r_{1}>0$ such that

$$
f(t, u)<\eta u
$$

for any $t \in J$, and $0<u \leq r_{1}$.

Let $D=\left\{w \in P:\left\|w+u^{*}\right\| \leq r_{1}\right\}$, where $u^{*}(t)=\bar{g} a\left(n_{1}+\right.$ $\left.m_{1} t\right)$ for $t \in J$. So $\bar{g} a n_{1} \leq u^{*}(t) \leq \bar{g} a\left(n_{1}+m_{1}\right)$ for $t \in J$.

Let $0 \leq a<r_{1} / \bar{g} a\left(n_{1}+m_{1}\right)$, then $D \neq \emptyset$.

For any $w \in D$, we have

$$
\begin{aligned}
0 & \leq T w(t)=\int_{0}^{1} H(t, s) f\left(s, w(s)+u^{*}(s)\right) d s \\
& \leq \eta \int_{0}^{1} \max _{t \in J} H(t, s)\left|w(s)+u^{*}(s)\right| d s \\
& \leq\left\|w+u^{*}\right\| \eta \cdot \frac{1}{\eta} \\
& \leq r_{1} .
\end{aligned}
$$

That is $T(D) \subset D$. By the Schauder fixed point theorem, we can get that $T$ has at least one fixed point on $D$. In view of Lemma $15, u=w+u^{*}$ is a positive solution of the boundary value problem (1).

(2) Since $\lim \sup _{u \rightarrow+\infty} \max _{t \in J}(f(t, u) / u)=f^{\infty}$, there exist constants $\bar{r}_{2}>0$ and $\epsilon>0$ such that

$$
f(t, u)<\left(f^{\infty}+\epsilon\right) u
$$

for any $t \in J$ and $u \geq \bar{r}_{2}$, where $\epsilon$ satisfies $f^{\infty}+\epsilon<\eta$.

Let $M=\max _{(t, u) \in J \times\left[0, \bar{r}_{2}\right]} f(t, u)$. We have

$$
f(t, u) \leq M+\left(f^{\infty}+\epsilon\right) u \text { for }(t, u) \in J \times[0,+\infty) .
$$

We choose $r_{2}>\max \left\{\bar{r}_{2}, \quad M /\left(\eta-\left(f^{\infty}+\epsilon\right)\right)\right\}$ and $D=$ $\left\{w \in P:\left\|w+u^{*}\right\| \leq r_{2}\right\}$, where $u^{*}(t)=\bar{g} a\left(n_{1}+m_{1} t\right)$ for $t \in J$. Then, for any $w \in D$,

$$
\begin{aligned}
0 & \leq T w(t) \\
& =\int_{0}^{1} H(t, s) f\left(s, w(s)+u^{*}(s)\right) d s \\
& \leq \int_{0}^{1} \max _{t \in J} H(t, s)\left(M+\left(f^{\infty}+\epsilon\right)\left|w(s)+u^{*}(s)\right|\right) d s \\
& \leq\left(M+\left\|w+u^{*}\right\|\left(f^{\infty}+\epsilon\right)\right) \cdot \frac{1}{\eta} \\
& \leq\left(\left(\eta-\left(f^{\infty}+\epsilon\right)\right) r_{2}+r_{2}\left(f^{\infty}+\epsilon\right)\right) \cdot \frac{1}{\eta} \\
& =r_{2} .
\end{aligned}
$$

That is $T(D) \subset D$. By the Schauder fixed point theorem, we can get that $T$ has at least one fixed point on $D$. In view of Lemma $15, u=w+u^{*}$ is a positive solution of the boundary value problem (1).

Theorem 22. Suppose that (H1), (H2), and (H3) hold. If $0 \leq$ $f^{0}<\eta$ and $f_{\infty}>\eta / \gamma^{2}$, then there exists a constant $a^{*} \epsilon$ $(0,+\infty)$ with the following properties.

(1) $a^{*}$ separates $[0,+\infty)$ into two disjoint subintervals $M=\left[0, a^{*}\right]$ and $N=\left(a^{*},+\infty\right)$.

(2) The boundary value problem (1) has at least two positive solutions for each $a \in\left(0, a^{*}\right)$, has at least one positive solution for $a=0$ and $a=a^{*}$, and does not have positive solutions for each $a \in N$.

Proof. We have the following four steps to prove the conclusions of Theorem 22.

Step 1. Let

$$
\begin{gathered}
\Lambda=\{a \in[0,+\infty): \text { The boundary value problem } \\
\text { has at least one positive solution }\} .
\end{gathered}
$$

By Theorem 21(1), we have $\Lambda \neq \emptyset$.

For each $\tilde{a} \in \Lambda$, denote

$$
A(\widetilde{a})=\{a: 0 \leq a \leq \widetilde{a}\} .
$$

Then, in view of Theorem 18, $A(\widetilde{a}) \subseteq \Lambda$ if and only if $\tilde{a} \in \Lambda$.

It follows that $\Lambda$ is a bounded set from $f_{\infty}>\eta / \gamma^{2}$ and Theorem 20(2).

Let $M=\bigcup_{\widetilde{a} \in \Lambda} A(\widetilde{a})$, then $M$ is a bounded set. Hence, the set $M$ has the supremum; we denote $a^{*}=\sup M$ and we have $a^{*}>0$ from Theorem 21 .

Step 2. We prove that the boundary value problem (1) has at least one positive solution when $a=a^{*}$.

Since $a^{*}=\sup M$, there exist $\left\{a_{k}\right\} \subset M$ and $a_{k}<a^{*}$ such that $a_{k} \rightarrow a^{*}$ as $k \rightarrow+\infty$. 
Let $u_{k}$ be a solution of the boundary value problem (1) with $a$ replaced by $a_{k}$. By Lemma 8 , the boundary value problem (1) with $a=a_{k}$ is equivalent to

$$
\begin{aligned}
u_{k}(t)= & \int_{0}^{1} H(t, s) f\left(s, u_{k}(s)\right) d s \\
& +\bar{g} a_{k}\left(n_{1}+m_{1} t\right), \quad k=1,2, \ldots
\end{aligned}
$$

In view of Lemma $9,\left\{u_{k}\right\}$ is uniformly bounded and equicontinuous. By Arzela-Ascoli, $\left\{u_{k}\right\}$ has a convergent subsequence; we also denote $\left\{u_{k_{m}}\right\}$, and $\left\{u_{k_{m}}\right\}$ converge to $u$.

By Lebesgue dominated convergence theorem, we can get

$$
u(t)=\int_{0}^{1} H(t, s) f(s, u(s)) d s+\bar{g} a^{*}\left(n_{1}+m_{1} t\right) .
$$

Hence, $u$ is a positive solution of the boundary value problem (1) with $a=a^{*}$.

Step 3. By Theorem 18, when $0 \leq a \leq a^{*}$, the boundary value problem (1) has at least one positive solution. That is, $M=$ $\left[0, a^{*}\right]$.

Let $N=\left(a^{*},+\infty\right)$. Then $a^{*}$ separates $[0,+\infty)$ into two disjoint subintervals $M=\left[0, a^{*}\right]$ and $N=\left(a^{*},+\infty\right)$; we have that the boundary value problem (1) has at least one positive solution for each $a \in M$ and does not have positive solutions for each $a \in N$.

Step 4 . We prove the boundary value problem (1) has at least two positive solutions when $a \in\left(0, a^{*}\right)$.

For each $a \in\left(0, a^{*}\right)$, there exist $\underline{a}, \bar{a} \in M$, such that $0<$ $a<a<\bar{a}$.

Let $\bar{u}$ be a solution of the boundary value problem (1) with $a$ replaced by $\bar{a}$. By Theorem 18 , the boundary value problem (1) has a positive solution $u_{1}$ with $u_{1} \leq \bar{u}$.

Similarly, let $u$ be a solution of the boundary value problem (1) with $\bar{a}$ replaced by $\underline{a}$ and $\underline{u} \leq u_{1}$. Hence, $\underline{u} \leq$ $u_{1} \leq \bar{u}$.

In fact, we can easily show that $\underline{u}<u_{1}<\bar{u}$ from $\underline{a}<a<\bar{a}$ and (H1).

Let $\alpha=\underline{u}$ and $\beta=\bar{u}$. We can easily verify $\alpha$ and $\beta$ are a lower solution and an upper solution of the boundary value problem (1), respectively, and $\alpha<\beta$.

We choose $\widehat{a} \in N$, then $a<a^{*}<\widehat{a}$.

Define $K:[a, \widehat{a}] \times P \rightarrow E$ by

$$
K(\kappa, u)=\bar{g} \kappa\left(n_{1}+m_{1} t\right)+\int_{0}^{1} H(t, s) f(s, u(s)) d s .
$$

Let

$$
F(t, u)= \begin{cases}f(t, \beta(t)), & u>\beta(t), \\ f(t, u), & \alpha(t) \leq u \leq \beta(t), \\ f(t, \alpha(t)), & u<\alpha(t),\end{cases}
$$

and define $\widehat{K}:[a, \widehat{a}] \times P \rightarrow E$ by

$$
\widehat{K}(\kappa, u)=\bar{g} \kappa\left(n_{1}+m_{1} t\right)+\int_{0}^{1} H(t, s) F(s, u(s)) d s .
$$

Similar to the proof of Theorem 17, we can prove $K$ and $\widehat{K}$ are completely continuous for each $\kappa \in[a, \widehat{a}]$. By Lemma $8, u$ is a positive solution of the boundary value problem (1) if and only if $u=K(a, u)$.

By Lemma 19, there exists a constant $\tau$ such that the fixed point $u$ of $K$ satisfies $\|u\|<\tau$ for each $\kappa \in[a, \widehat{a}]$.

Let

$$
\Omega=\{u \in P:\|u\|<\tau, \alpha(t)<u<\beta(t), t \in J\} .
$$

Clearly, $\Omega$ is a nonempty open-bounded subset of $P$.

Since $F$ is bounded, there exists a constant $R>\tau>0$ such that $|\widehat{K}(\kappa, u)|<R$ for any $(\kappa, u) \in[a, \widehat{a}] \times P$. Let $B(\theta, R)=\{u \in E:\|u\|<R\}$; it is obvious that $\Omega \subset P \cap B(\theta, R)$ and $u \neq \mu \widehat{K} u$ for $u \in P \cap \partial B(\theta, R)$, and $\mu \in[0,1]$. Hence, by Lemma 10 , for each $\kappa \in[a, \widehat{a}]$, we have

$$
i(\widehat{K}(\kappa, u), P \cap B(\theta, R), P)=1 .
$$

Because $\widehat{K}$ does not have a fixed point on $P \cap(\overline{B(\theta, R)} \backslash \Omega)$, then for each $\kappa \in[a, \widehat{a}]$,

$$
i(\widehat{K}(\kappa, u), P \cap(B(\theta, R) \backslash \bar{\Omega}), P)=0 .
$$

We notice that $\left.\widehat{K}\right|_{\Omega}=K$, by the excision property of the fixed point index, (106) and (107), for each $\kappa \in[a, \widehat{a}]$, we have

$$
\begin{aligned}
i(K & (\kappa, u), P \cap \Omega, P) \\
= & i(\widehat{K}(\kappa, u), P \cap B(\theta, R), P) \\
& -i(\widehat{K}(\kappa, u), P \cap(B(\theta, R) \backslash \bar{\Omega}), P)=1 .
\end{aligned}
$$

Since $\widehat{a} \in N$, we have $K(\widehat{a}, u) \neq u$ for any $u \in P$. So,

$$
i(K(\widehat{a}, u), P \cap B(\theta, R), P)=0 .
$$

We define $H:[0,1] \times P \cap \overline{B(\theta, R)} \rightarrow E$ by

$$
H(t, u)=K((1-t) a+t \widehat{a}, u) .
$$

It is obvious that $H$ is completely continuous.

We can prove $H(t, u) \neq u$ for $(t, u) \in[0,1] \times P \cap \partial B(\theta, R)$.

Otherwise, if there exists $\left(t_{0}, u_{0}\right) \in[0,1] \times P \cap \partial B(\theta, R)$ such that $H\left(t_{0}, u_{0}\right)=u_{0}$, that is

$$
K\left(\left(1-t_{0}\right) a+t_{0} \widehat{a}, u_{0}\right)=u_{0}, \quad\left\|u_{0}\right\|=R .
$$

Hence, $u_{0}$ is a solution of the boundary value problem (1) with $a$ replaced by $\kappa=\left(1-t_{0}\right) a+t_{0} \widehat{a}$. We can get $\left\|u_{0}\right\|<\tau$, which is a contradiction.

According to the homotopy invariance of the fixed point index and (109), it follows that

$$
\begin{aligned}
i( & K(a, u), P \cap B(\theta, R), P) \\
& =i(H(0, u), P \cap B(\theta, R), P) \\
& =i(H(1, u), P \cap B(\theta, R), P) \\
& =i(K(\widehat{a}, u), P \cap B(\theta, R), P)=0 .
\end{aligned}
$$


By the additivity property of the fixed point index, (108) and (112), we obtain

$$
i(K(a, u), P \cap B(\theta, R) \backslash \bar{\Omega}, P)=-1 .
$$

Therefore, the boundary value problem (1) has a solution $u_{2}$ in $P \cap(B(\theta, R) \backslash \bar{\Omega})$ and $u_{1} \neq u_{2}$ from $u_{1} \in \Omega$. That is, the boundary value problem (1) has at least two positive solutions.

\section{Conflict of Interests}

The authors declare that there is no conflict of interests regarding the publication of this paper.

\section{Acknowledgments}

The authors are grateful to the referees for their valuable comments and suggestions. This work is supported by the National Natural Science Foundation of China (no. 11171220) and the Foundation of the Education Department of Shanghai (no. 10ZZ93).

\section{References}

[1] S. G. Samko, A. A. Kilbas, and O. I. Marichev, Fractional Integrals and Derivatives, Gordon and Breach Science Publishers, Yverdon, Switzerland, 1993.

[2] A. A. Kilbas, H. M. Srivastava, and J. J. Trujillo, Theory and Applications of Fractional Differential Equations, vol. 204, Elsevier Science, Amsterdam, The Netherlands, 2006.

[3] K. Diethelm, The Analysis of Fractional Differential Equations, vol. 2004, Springer, Berlin, Germany, 2010.

[4] K. Diethelm and A. D. Freed, "On the solution of nonlinear fractional order differential equations used in the modeling of viscoplasticity," in Science Computing in Chemical EngineeringComputational Fluid Dynamics, Reaction Engineering and Molecular Properties, F. Keil, W. Mackens, H. Voss, and J. Werther, Eds., pp. 217-224, Springer, Heidelberg, Germany, 1999.

[5] R. Hilfer, Applications of Fractional Calculus in Physics, World Scientific, Singapore, 2000.

[6] V. Lakshmikantham, S. Leela, and J. Vasundhara, Theory of Fractional Dynamic Systems, Cambridge Academic Publisher, Cambridge, UK, 2009.

[7] I. Podlubny, Fractional Ddifferential Equations, vol. 198, Academic Press, New York, NY, USA, 1999.

[8] H. Schiessel, R. Metzler, A. Blumen, and T. F. Nonnenmacher, "Generalized viscoelastic models: their fractional equations with solutions," Journal of Physics A, vol. 28, no. 23, article 012, pp. 6567-6584, 1995.

[9] R. W. Ibrahim and M. Darus, "Subordination and superordination for univalent solutions for fractional differential equations," Journal of Mathematical Analysis and Applications, vol. 345, no. 2, pp. 871-879, 2008.

[10] S. Ladaci, J. J. Loiseau, and A. Charef, "Fractional order adaptive high-gain controllers for a class of linear systems," Communications in Nonlinear Science and Numerical Simulation, vol. 13, no. 4, pp. 707-714, 2008.
[11] J. Wang, Y. Zhou, W. Wei, and H. Xu, "Nonlocal problems for fractional integrodifferential equations via fractional operators and optimal controls," Computers \& Mathematics with Applications, vol. 62, no. 3, pp. 1427-1441, 2011.

[12] Y. Xu and Z. He, "The short memory principle for solving Abel differential equation of fractional order," Computers \& Mathematics with Applications, vol. 62, no. 12, pp. 4796-4805, 2011.

[13] J. P. Keener, Principles of Applied Mathematics, Addison-Wesley, Redwood City, Calif, USA, 1988.

[14] G. H. Meyer, Initial Value Methods for Boundary Value Problems, Academic Press, New York, NY, USA, 1973.

[15] L. H. Thomas, "The calculation of atomic fields," Proceedings of the Cambridge Philosophical Society, vol. 23, no. 5, pp. 542-548, 1927.

[16] J. R. Esteban and J. L. Vázquez, "On the equation of turbulent filtration in one-dimensional porous media," Nonlinear Analysis: Theory, Methods \& Applications, vol. 10, no. 11, pp. 1303-1325, 1986.

[17] A. A. Kilbas and S. A. Marzan, "Nonlinear differential equations with the Caputo fractional derivative in the space of continuously differentiable functions," Journal of Differential Equations, vol. 41, no. 1, pp. 82-89, 2005.

[18] S. Zhang, "Positive solutions for boundary-value problems of nonlinear fractional differential equations," Electronic Journal of Differential Equations, vol. 2006, no. 36, pp. 1-12, 2006.

[19] X. Liu, M. Jia, and B. Wu, "Existence and uniqueness of solution for fractional differential equations with integral boundary conditions," Electronic Journal of Qualitative Theory of Differential Equations, no. 69, pp. 1-10, 2009.

[20] M. Jia and X. Liu, "Three nonnegative solutions for fractional differential equations with integral boundary conditions," Computers \& Mathematics with Applications, vol. 62, no. 3, pp. 14051412, 2011.

[21] X. Liu and M. Jia, "Multiple solutions for fractional differential equations with nonlinear boundary conditions," Computers \& Mathematics with Applications, vol. 59, no. 8, pp. 2880-2886, 2010.

[22] S. Z. Rida, H. M. El-Sherbiny, and A. A. M. Arafa, "On the solution of the fractional nonlinear Schrödinger equation," Physics Letters A, vol. 372, no. 5, pp. 553-558, 2008.

[23] J. H. Barrett, "Differential equations of non-integer order," Canadian Journal of Mathematics, vol. 6, pp. 529-541, 1954.

[24] M. Al-Refai and M. A. Hajji, "Monotone iterative sequences for nonlinear boundary value problems of fractional order," Nonlinear Analysis: Theory, Methods \& Applications, vol. 74, no. 11, pp. 3531-3539, 2011.

[25] L. Lin, X. Liu, and H. Fang, "Method of upper and lower solutions for fractional differential equations," Electronic Journal of Differential Equations, vol. 2012, no. 100, pp. 1-13, 2012.

[26] Z. Bai and H. Lü, "Positive solutions for boundary value problem of nonlinear fractional differential equation," Journal of Mathematical Analysis and Applications, vol. 311, no. 2, pp. 495-505, 2005.

[27] Z. Bai and T. Qiu, "Existence of positive solution for singular fractional differential equation," Applied Mathematics and Computation, vol. 215, no. 7, pp. 2761-2767, 2009.

[28] D. Jiang and C. Yuan, "The positive properties of the Green function for Dirichlet-type boundary value problems of nonlinear fractional differential equations and its application," Nonlinear Analysis: Theory, Methods \& Applications, vol. 72, no. 2, pp. 710-719, 2010. 
[29] C. F. Li, X. N. Luo, and Y. Zhou, "Existence of positive solutions of the boundary value problem for nonlinear fractional differential equations," Computers \& Mathematics with Applications, vol. 59, no. 3, pp. 1363-1375, 2010.

[30] J. R. L. Webb and G. Infante, "Positive solutions of nonlocal boundary value problems involving integral conditions," NoDEA. Nonlinear Differential Equations and Applications, vol. 15, no. 1-2, pp. 45-67, 2008.

[31] J. R. L. Webb and G. Infante, "Positive solutions of nonlocal boundary value problems: a unified approach," Journal of the London Mathematical Society, vol. 74, no. 3, pp. 673-693, 2006.

[32] Z. Yang, "Positive solutions to a system of second-order nonlocal boundary value problems," Nonlinear Analysis: Theory, Methods \& Applications, vol. 62, no. 7, pp. 1251-1265, 2005.

[33] M. Feng, D. Ji, and W. Ge, "Positive solutions for a class of boundary-value problem with integral boundary conditions in Banach spaces," Journal of Computational and Applied Mathematics, vol. 222, no. 2, pp. 351-363, 2008.

[34] S. Xi, M. Jia, and H. Ji, "Multiple nonnegative solutions for second-order boundary-value problems with sign-changing nonlinearities," Electronic Journal of Differential Equations, vol. 2009, no. 66, pp. 1-10, 2009.

[35] L. Kong and Q. Kong, "Second-order boundary value problems with nonhomogeneous boundary conditions. I," Mathematische Nachrichten, vol. 278, no. 1-2, pp. 173-193, 2005.

[36] L. Kong and Q. Kong, "Second-order boundary value problems with nonhomogeneous boundary conditions. II," Journal of Mathematical Analysis and Applications, vol. 330, no. 2, pp. 1393-1411, 2007.

[37] L. Kong and Q. Kong, "Higher order boundary value problems with nonhomogeneous boundary conditions," Nonlinear Analysis: Theory, Methods \& Applications, vol. 72, no. 1, pp. 240-261, 2010.

[38] D. J. Guo and V. Lakshmikantham, Nonlinear Problems in Abstract Cones, vol. 5, Academic Press, New York, NY, USA, 1988.

[39] K. Deimling, Nonlinear Functional Analysis, Springer, Berlin, Germany, 1984. 


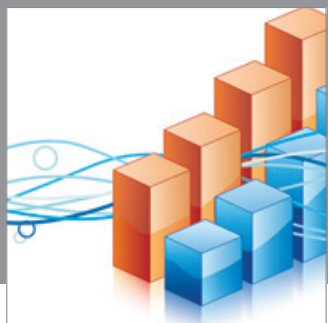

Advances in

Operations Research

mansans

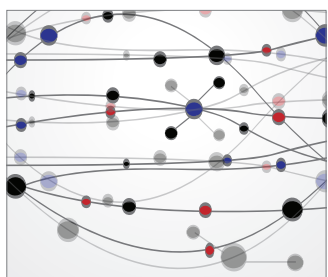

The Scientific World Journal
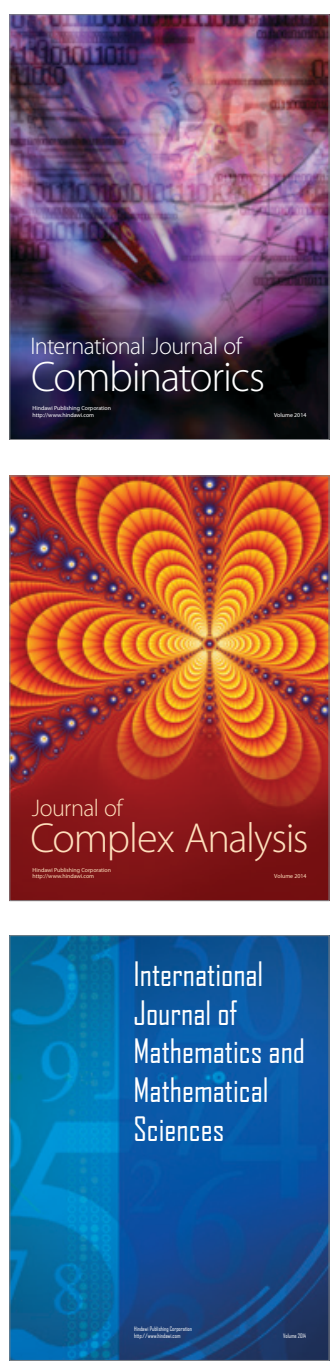


Submit your manuscripts at http://www.hindawi.com
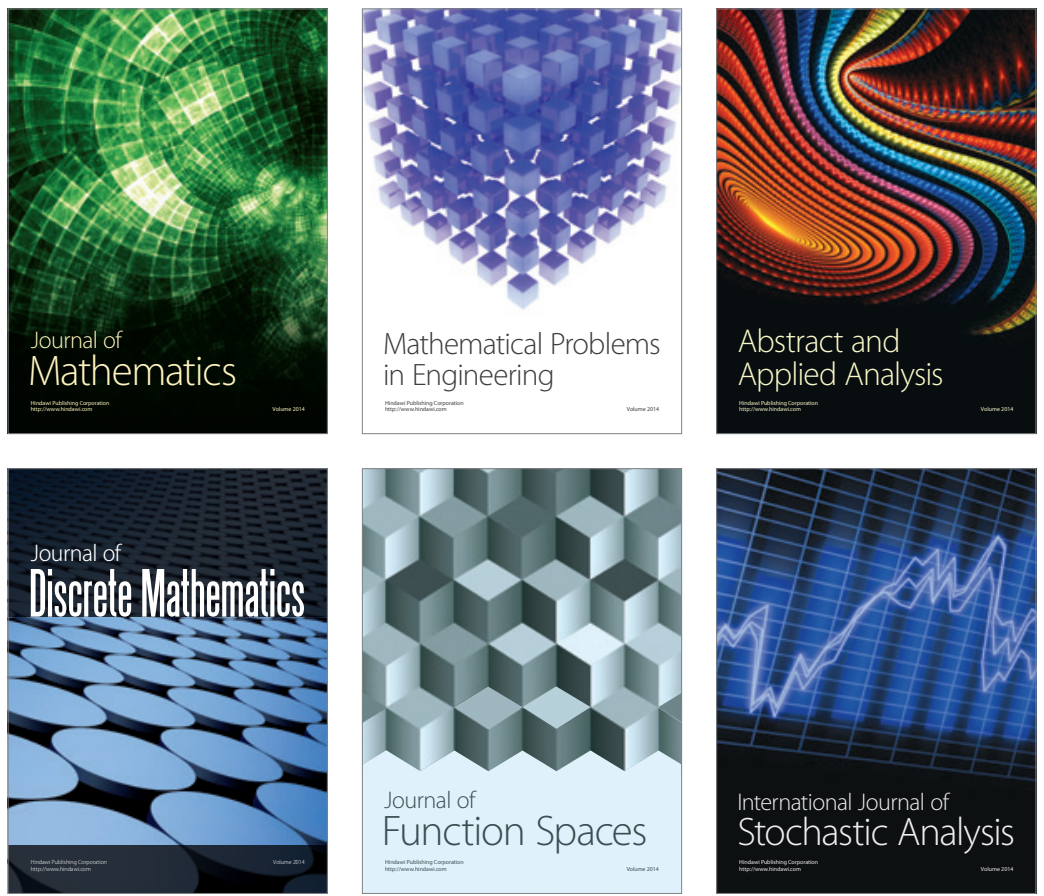

Journal of

Function Spaces

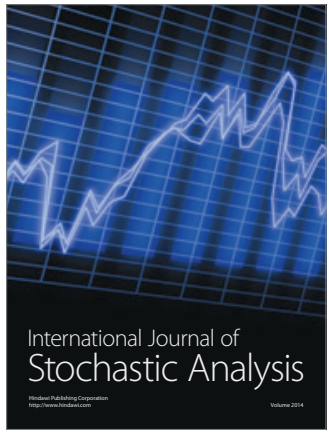

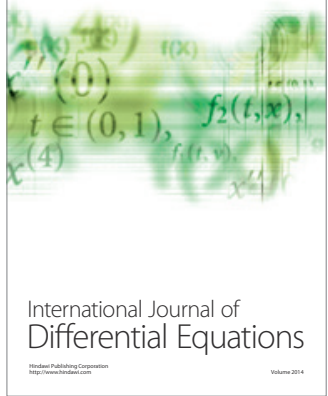
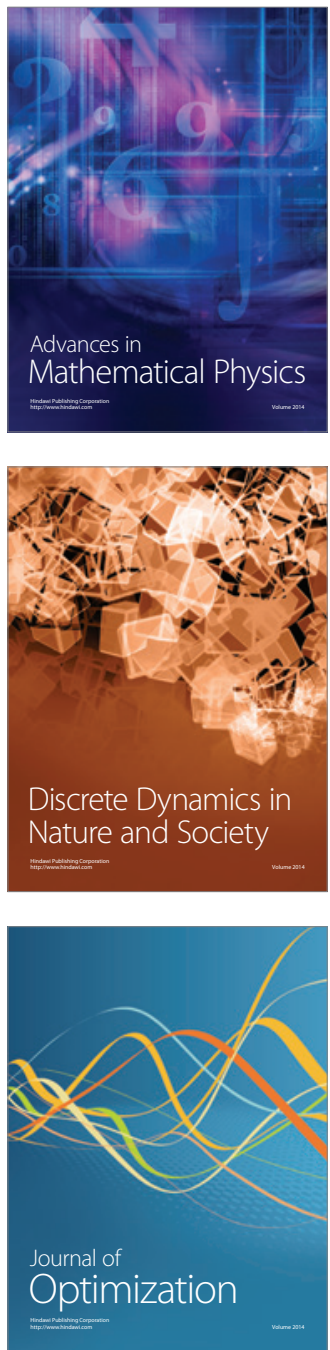